PRODUCTION

ENGINEERING ARCHIVES
2014, Vol. 5, No 4, pp 42-46

ISSN 2353-5156

ISSN 2353-7779 (print version)

(online version)

Article history: Received: 10.10 .2014

Accepted: 23.10.2014

Online: 31.12 .2014

Available online on: http://www.qpij.pl

Exist since $4^{\text {th }}$ quarter 2013

\title{
Investigation of dynamic characteristics of the scrubber based dimensional model in the software package ANSYS
}

\author{
Regina Usmanova ${ }^{1}$, Dorota Klimecka-Tatar ${ }^{2}$, Gennady Zaikov ${ }^{3}$ \\ ${ }^{1}$ Ufa State technical university of aviation 12 Karl Marks str., 450000, Ufa, Bashkortostan, Russia \\ ${ }^{2}$ Institute of Production Engineering, Czestochowa University of Technology, Czestochowa, Poland \\ ${ }^{3}$ N.M. Emanuel Institute of Biochemical Physics, Russian academy of sciences, 4 Kosygin str., 119334, Moscow, Russia
}

\begin{abstract}
This paper deals with the mathematical modeling of the separation process in the dynamic scrubber with the generation of the model in Ansys CFX software. A model of gas flow and dispersion medium and the hydrodynamic picture of a swirling movements phases is obtained. The conducted research provide an opportunity to predict aerohydrodynamic characteristics of the device at the design stage. Doing so may provide design solutions to such units of the apparatus that could dramatically improve the efficiency of gas cleaning.
\end{abstract}

Key words - computational domain; dynamic scrubber; swirler; hydraulic resistance; line a current

\section{Introduction}

The possibilities of a tangential inlet gas into the unit and the formation of internal swirling flows are extremely diverse. Vortex gas flow is a complex form of movement entirely dependent on the design parameters and tightening devices. These devices determine the aerodynamic characteristics and flow chambers: the degree of furling, hydraulic resistance, structure and uneven speed, features recirculation zones, injection capacity, turbulence intensity. However, despite the differences of known devices in design, size and purpose formed in these gas streams, they have common patterns.

Currently mathematical models of gas purification are used formed on simplified theoretical concepts of gas flow. They do not sufficiently take into account the operational and design parameters of gas cleaning devices, as well as aero-hydrodynamic properties of gas-dispersed flows. These models can not be used to search for the best options of integrated gas cleaning systems, as they show the properties of objects in a narrow range of parameters. More complete and appropriate mathematical models are needed based on the study of the aerodynamics of gas taking place in these processes.

The use of computer technology and software is vital in order to compute the hydrodynamic characteristics of eddy currents during the development and design of industrial devices. This avoids the need for costly field tests of gas purification apparatus.

Software suite is a modern ANSYS-14/CFXmodeling tool, based on the numerical solution of the equations of hydrodynamics (KAPLUN A.B. et al. 2003, 
BASOW K.A. 2005). Hydrodynamic calculation makes it possible to determine the flow resistance of the device and to predict the efficiency of the separation process in the design stage.

\section{Statement of the problem}

Mathematical modeling is always based on some physical hypothesis that simplifies considered real objects. At this stage of the development of mathematics it is possible not only to describe the physical model in the form of equations and additional conditions, but also to solve the problem. For the formulation of the problem of modeling and the subsequent investigation of the processes occurring in the vortex of centrifugal machines, you need to define the relationship between the parameters of the device.

In studies of vortex centrifugal devices primarily include spatial flow. Similarly it is conducted in models based on the hypothesis of a plane vortex. Motion of the gas is described by the Navier-Stokes equations. The equation is introduced with the closure of the fluctuating components of the hypothesis on the path of displacement. The values of the tangential and radial velocity are taken close to each other. Axial velocity is very small. Exclusion from consideration of the axial movement of the gas is greatly reduced, the (idealized). This is not consistent with the physical picture, in which a large place is occupied by forward and backward axial currents. The results of these studies are of interest to determine the effects of the vortex flow asymmetry with respect to the axis of the camera. Numerical analysis of the gas inside the dynamic scrubber reduces to solving the Navier-Stokes equations (Patent 2339435 RF, B01 D47/06). A standard $(\mathrm{k}-\varepsilon)$-turbulence model is used. To find the scalar parameters $\mathrm{k}$ and $\varepsilon$ are two additional model equations containing empirical constants (BULGAKOV V.K., POTAPOV I.I. 2003, GONCHAROV A.L., FryazINOV I.V. 1986, TRINH C.M. 1998). The computational grid was built in the grid generator ANSYS ICEM CFD. The grid consists of 1247542 elements.

The grid was constructed on a uniform and nonuniform radius along the axial co-ordinate. Unevenness wondered law exponentially was decreasing to the exhaust pipe and increasing the bottom unit. Denomi- nators geometric progressions were chosen so that in the high-velocity gradient mesh was as thick as possible. On the input and output sections of laws of changing the current function, on the axis of the unit gradient target variable were constant. The conical part determines the boundary conditions and restrictions on the size of the computational grid, i.e. the reduction of radius corresponds to a decrease of the boundary node. A general view of the computational domain is shown in Figure 1.

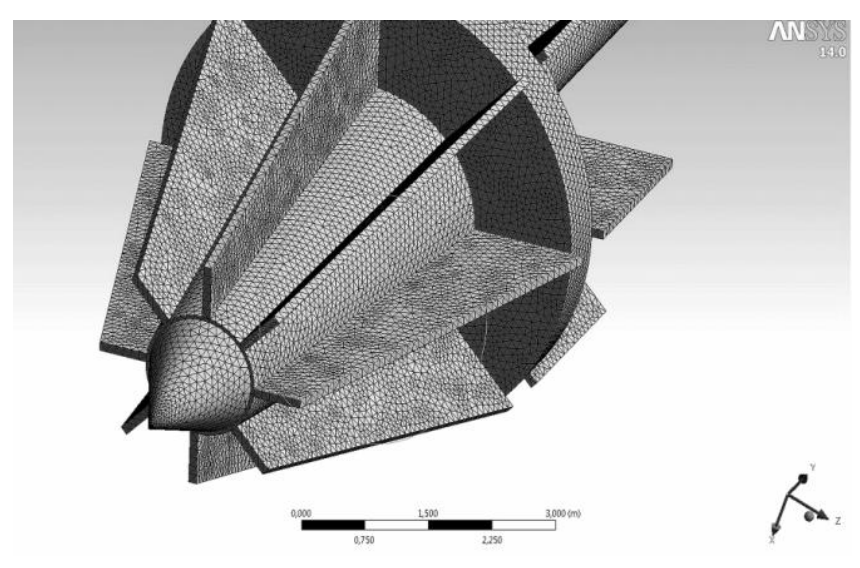

Fig. 1.General view of the computational domain.

\section{Analysis of the results}

The flow of gas and dust in a dynamic scrubber is complex. This is explained by the fact that in the central part of the device is a vane swirler. Analysis of fluid flow and distribution of deposited particles in a dynamic scrubber showed that the presence of turbulent diffusion particles concentrate at the vessel wall is not a thick layer, and in the form of gas and dust loosened concentrated layer.

In this case, the dust is localized in the annular wall layer of a certain thickness in a spiral of dust accumulations in the form of bundles. This initiated the formation of helical bundles in a dusty swirl vane. Alongside the passage of dust in vane curved channel is the concentration of particles in the peripheral zone of the channel. Thus, after the passage of a uniform flow swirler vane is divided into a number of parallel streams with alternating then small, then large concentration of dust. The thickness and density of the boundary layer depends on the gas velocity, the angle 
of twist, the character input stream to a dynamic scrubber. Higher speeds may reduce the thickness of the boundary layer, in spite of increasing the role of turbulent diffusion.

Reducing the length of the inlet pipe reduces the eccentricity of the axis of rotation of the gas flow from the geometrical axis of the apparatus. Even in this case, the center of the rotational flow does not coincide completely with the geometric axis of the machine. There is some minor eccentricity, the value of which does not exceed $6 \div 8 \%$ of the radius of the device. There is also research about the presence of such eccentricity swirling flow (Sazhin B.S., Akulich A.V. 2001).

Given that the eccentricity of the machine is small, and in its central part of the irrigation device, we consider for a gas flow as symmetric about the axis of the machine.

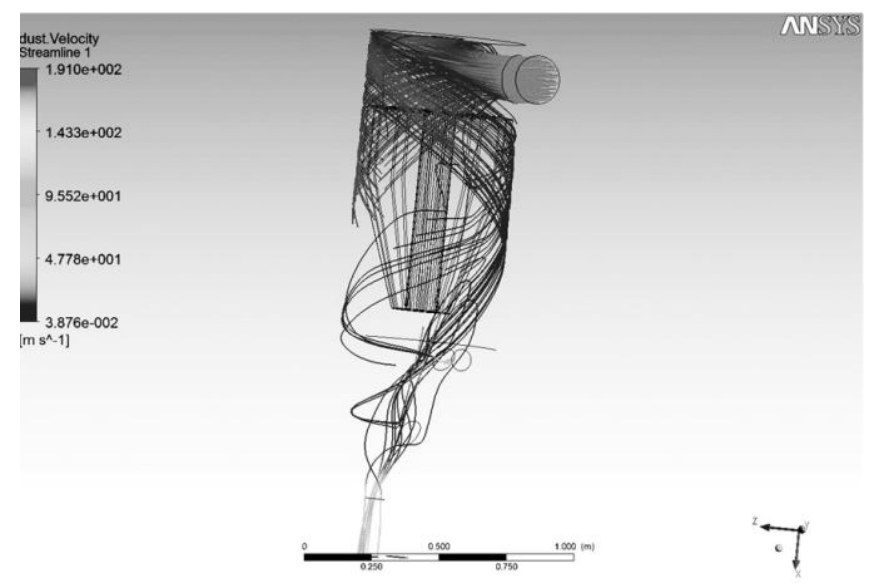

Fig. 2. The flow pattern of gas and dust in the dynamic flow scrubber.

\section{Output calculation formulas}

Aerodynamics of the apparatus were conducted in the range of the Reynolds number changes from $3,5^{\cdot} 10^{4}$ to $15^{\cdot} 10^{4}$. This corresponds to an average of 5 to $25 \mathrm{mps}$. The degree of spin flow was constant at $K=1,5$.

A large study focused on determination of the resistance unit and study of the effect of dynamic geometry scrubber on the energy characteristics $\triangle P$ and $\xi$ (USMANOVA R.R. et al. 2003, SAZHIN B.S., AKULICH A.V.2001). Resistance scrubber is calculated by the total pressure drop at the entrance to the unit and to exit. In this case, the Bernoulli equation for incompressible gas is written in the form of:

$$
\rho \frac{\alpha_{k} W_{1}^{2}}{2}+P_{1}+\rho_{g} g z_{1}=\rho_{g} \frac{\alpha_{k} W_{2}^{2}}{2}+P_{2}+\Delta P
$$

where $z$ - the distance between the sections; $P_{l}$, $P_{2}$ - Static pressure, Pa; $W_{l}, W_{2}$ - the average flow velocity in the annulus and in the exhaust pipe; $\alpha_{k}$ Coriolis coefficient taking into account the nonuniformity of the velocity distribution in the cross section. Ratio is the ratio of the true kinetic energy to kinetic energy of the flow, calculated at the average rate (for the turbulent regime of motion take $\alpha=$ $1,05 \div 1,10)$. Knowing $\Delta P$, we can calculate the coefficient of hydraulic resistance, referred to the conditional mean in terms of the speed machine $W_{0}$.

$$
\zeta_{0}=\frac{2 \Delta P}{\rho_{g} W_{0}^{2}}
$$

Hydraulic resistance of centrifugal machines are generally viewed as the local resistance. Hydraulic resistance co-efficient, pressure losses in the unit determined experimentally and are mainly as a function of the geometry and the Reynolds number (IDELCHIK I.E. 1954).

IDELCHIK I.E. 1954, TARASOVA L.A. et. al. 2004 show an approach to the calculation of flow resistance as the sum of the individual parts of resistance tract. This approach helps to clarify the physical nature of the process, to evaluate different design aerodynamic perfection swirlers.

Hydraulic resistance machines centrifugal type represented by the sum of the resistances of the cylindrical device, swirler and exhaust pipe. The processing of the experimental data suggested an empirical equation for calculating the co-efficient of hydraulic resistance machines centrifugal type.

It was found that the flow resistance of the "dry" machine obeys the square of the velocity of the gas. With the increase in the co-efficient of spin $\xi$ down. This is due to the decreasing level of the tangential component of the gas velocity in the swirler.

At some value of $K$, the co-efficient of hydraulic resistance is almost independent of the flow scrubbing 
liquid. This is explained by the influence of two factors related to the supply of irrigating fluid dynamic scrubber. On the one hand - increasing $\xi$ due to the increase of pressure loss of the gas stream to transport liquids; while on the other hand - reducing $\xi$ due to the decrease of the tangential velocity of the gas by the braking action of the liquid.

On this basis an empirical mathematical model for calculating the co-efficient of hydraulic esistance was constructed, including a formula to calculate $\xi$ «dry» machine:

$$
\xi_{\mathrm{dry}}=\frac{1}{n}\left(\left(R_{m}\right)^{2 n}-1\right)+\frac{\alpha}{K^{2}} \cdot\left(\frac{\vartheta_{\text {out }}}{\vartheta_{\text {in }}}\right)^{2}
$$

Empirical relationship for calculating the pressure drop in the gas transport liquid:

$$
\xi_{t r}=4 \cdot\left(\frac{\mathrm{Q}}{G}\right)^{0,4} \cdot \sqrt{1+\frac{1}{K^{2}}}
$$

and ultimate dependence for calculating $\xi$ irrigation apparatus

$$
\begin{aligned}
& \xi_{\text {irr }}=\frac{1}{n}\left(\left(R_{m}\right)^{2 n}-1\right)+\alpha \frac{\varepsilon^{2}}{K^{2}} \cdot\left(1+\frac{\rho_{l}}{\rho_{g}}\right) \cdot\left(\frac{\vartheta_{\text {out }}}{\vartheta_{\text {in }}}\right)^{2}+ \\
& +4\left(\frac{\mathrm{Q}}{G}\right)^{0,4} \cdot \sqrt{1+\frac{1}{K^{2}}}
\end{aligned}
$$

where $\xi$-co-efficient of hydraulic resistance; $R_{m}-$ radius of the cylindrical chamber, $\mathrm{m} ; \rho_{n}, \rho_{l}-$ density of gas and liquid, $\mathrm{kg} / \mathrm{m}^{3} ; \mathrm{v}_{\text {in }} \mathrm{v}_{\text {ou }}$ - velocity of gas at the inlet and outlet of the unit, mps; $n, \varepsilon$-indicators vortex movement, $K$ - factor twist swirler; $Q, G-$ liquid and gas flow $\mathrm{kg} / \mathrm{m}^{3} ; \alpha-$ twist angle of the flow, ${ }^{\circ}$.

The resulting formula takes into account the presence of the dispersed phase and the partial loss of swirling flow.

\section{Processing of results}

Figure 3 shows that the swirl angle of the blades with $\alpha=45^{\circ}$ has the lowest power characteristics. However, the efficiency in the gas cleaning unit with swirler is reduced by $6 \div 8 \%$ in comparison with the unit, where the blades are tilted at an angle $\alpha=30^{\circ}$. This can be explained by the decreasing flow swirling, which is characterized by a relative twist angle $\left(90^{\circ} \div\right.$ $45^{\circ}$ ), and the lowest centrifugal forces.

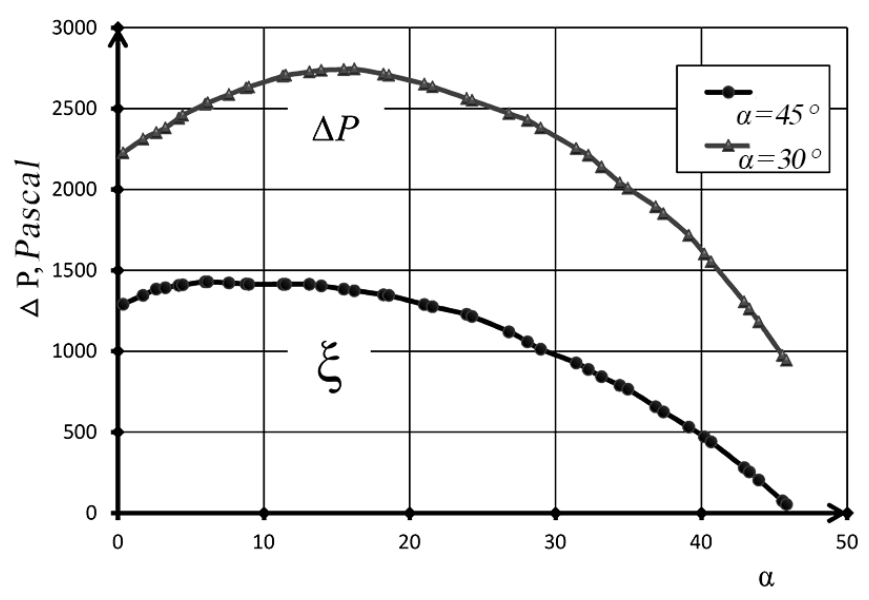

Fig. 3. The impact of the angle $\alpha$ on the energy characteristics of dynamic scrubber.

A study of hydrodynamic characteristics scrubber showed that the co-efficient of hydraulic resistance depends strongly on the angle of the blade swirler $\alpha$. It also depends on the movement of gas-dispersed medium defined by the Reynolds number $R e=\rho D v / \mu$. As seen in Figure 4, with increasing Reynolds number of $8 \cdot 10^{4}$ sets scaling of $\xi$. The exception is with the swirl angle of blades $35,5^{\circ}$, installation of which continues to increase hydraulic resistance.

Therefore, from the point of view of increasing the efficiency of gas treatment, preference should be given to the greatest Swirl swirled flow $-30^{\circ}$. 


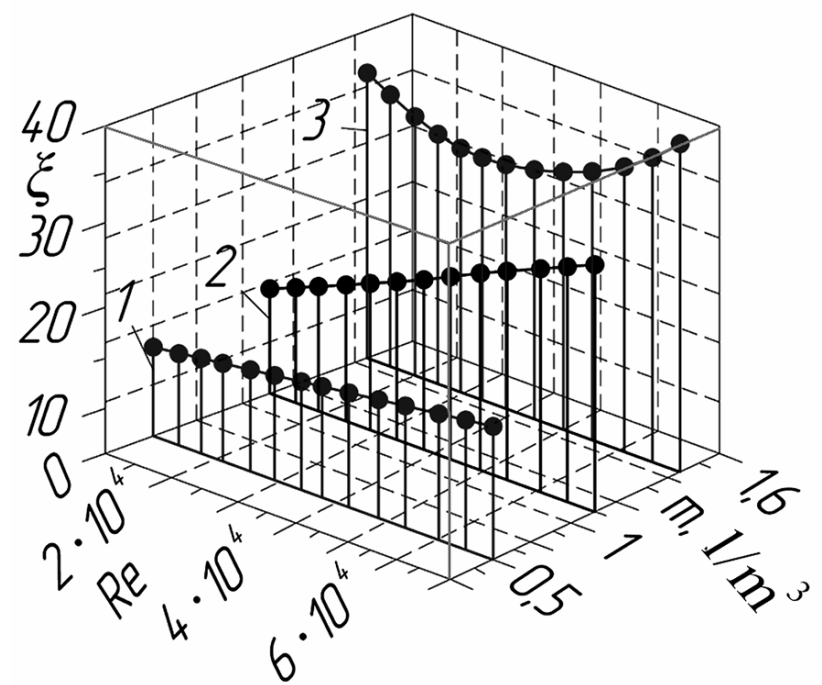

Fig. 4. The dependence of the hydraulic resistance of the Reynolds number and the angle of the blades of the swirler.

\section{Conclusion}

1. The program ANSYS-14/CFX used mathematical model of motion of polydispersed gas system. The character of the movement of dust particles under the influence of centrifugal force allowed to choose the desired hydrodynamic conditions and taking into account the design under various conditions of scrubber.

2. Experimental study of the hydrodynamic characteristics of the device to determine the empirical constants and test the adequacy of the hydrodynamic model.

3. The empirical relationships for determining the co-efficient of hydraulic resistance scrubber. They take into account the diameter of the separation chamber, the gas density and the scrubbing liquid, the angle of the blades of the swirler. The dependences obtained are suitable for the calculation of centrifugal machines of any type.

\section{References}

1. KAPLUN A.B. et al. 2003. ANSYS in the hands of the engineer. A practical guide. Moscow VNIIMP 272.

2. BASOW K.A. 2005. ANSYS and LMS Virtual Lab. Geometric Modeling. M.: DMK Press, p. 640.

3. Patent $2339435 \mathrm{RF}, \mathrm{B01}$ D47/06 dynamically scrubber. R.R. Usmanova. - Published 27.11.2008. Bull. № 33.
4. Bulgakov V.K., I.I. Potapov. 2003. Finite element scheme of the High-order for the Navier-Stokes equations. Modified by the SUPG-method. Sat. proceedings of the 16th International Conference "Mathematical Methods in Engineering and Technology". V.1., St. Petersburg. University Press, 2003.

5. Goncharov A.L. FryAZINOV I.V. 1986. On the construction of monotone of difference schemes for the Navier-Stokes equations on devyatito of point patterns. Institute of Applied Mathematics. Keldysh RAN.M. 93, p.14-16.

6. TRINH C.M. 1998. Turbulence Modeling of Confined Swirling Flows. Roskilde, Riso National Laboratory. Riso-R-647(EN).

7. SAZHIN B.S. AKULICH A.V. 2001. Mathematical modeling of the gas in the separation zone of the oncethrough vortex apparatus based on the model of turbulence. Theoretical Foundations of Chemical Engineering. 35(5), p. 472 - 478.

8. IDELCHIK I.E. 1954. Hydraulic resistance of a (physical-mechanical basis) p. 316.

9. TARASOVA L.A et.al. 2004. Hydraulic calculation of the resistance of the vortex unit. Chemical and Petroleum mechanical engineering. 2, p.11-12. 